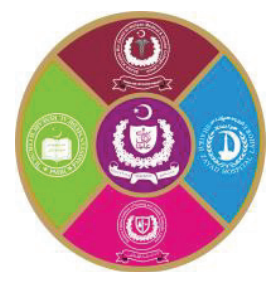

\title{
Histological Study of Commiphora mukul, Withania somnifera and Thyroxine in a Murine Model of Hypothyroidism
}

${ }^{1}$ Tehreem Zulfiqar, ${ }^{2}$ Saadia Shahzad Alam, ${ }^{1}$ Abdul Jabbar, ${ }^{3}$ Mariyam Iftikhar

${ }^{1}$ Department of Pharmacology, Nishtar Medical University, Multan

${ }^{2}$ Department of Pharmacology, Shaikh Zayed Medical Complex, Lahore.

${ }^{3}$ Department of Pharmacology, Akhtar Saeed Medical and Dental College, Lahore.

\begin{abstract}
Introduction: Hypothyroidism is a common endocrine disorder not only in developing countries like Pakistan but also in the developed countries having a prevalence of about $4-5 \%$. Thyroxine remains the gold standard treatment for hypothyroidism but due to its adverse effects, newer and safer phyto alternatives are the need of hour. Aims \& Objectives: Present study was designed to establish effects of Commiphora mukul, and Withania somnifera on thyroid histology in methimazole induced hypothyroidism in order to compare the effects of these plants on thyroid histology with thyroxine. Place and duration of study: This experimental study was carried out in the animal house of PGMI, Lahore. Total duration of study was 38 days. Material \& Methods: 60 rats were sorted in 6 groups with 10 animals each. G-1 was the control group. G-2 received methimazole $60 \mathrm{mg} / \mathrm{kg} /$ day for 21 days to induce hypothyroidism and left untreated for self recovery \& G3-6 were induced with methimazole in the same way. After induction G3-6 were treated for 15 days except group 2, G-3: Commiphora mukul Ethanolic Extract (CMEE) $0.2 \mathrm{~g} / \mathrm{kg}$ /day orally, G4: Withania somnifera Ethanolic Extract (WSEE) $1.4 \mathrm{~g} / \mathrm{kg} /$ day orally. G-5: CMEE $0.2 \mathrm{~g} / \mathrm{kg} / \mathrm{day}+$ WSEE $1.4 \mathrm{~g} / \mathrm{kg} /$ day orally. G-6 received the gold standard Thyroxin $0.6 \mathrm{ug} / 100 \mathrm{gm}$ BW S/C. Thyroid was excised for histology at the end i.e at day 38 . Results: Improved thyroid follicular morphology was seen in all treatment groups in their epithelium, colloid content, reversion of chromatin to normal and decreased nuclear pyknosis. However both extract CMEE \& WSEE in combination exhibited equi to greater potential than thyroxine $0.6 \mu \mathrm{g} / 100 \mathrm{gm}$ BW alone. Conclusion: So our study shows that treatment with combination of CMEE $0.2 \mathrm{~g} / \mathrm{kg}+$ WSEE $1.4 \mathrm{~g} / \mathrm{kg}$ orally for 15 days improved thyroid histology significantly which is comparable to gold standard thyroxine proving the potential of this combination to substitute thyroxine.
\end{abstract}

Key words: Methimazole, Hypothyroidism, Commiphora mukul, Withania somnifera, Thyroxine

\section{INTRODUCTION}

$\mathrm{H}_{3}$ ypothyroidism, characterized by reduced functioning of the thyroid gland which ultimately causes generalized slowing of all the bodily functions is posing a major health problem in both the developing as well as the developed world. ${ }^{1}$ Its prevalence in developed world is $4-5 \% .^{2}$ In developing countries like Pakistan, prevalence of primary hypothyroidism is found to be $63.3 \%$ which is a significant figure. ${ }^{3}$ Methimazole (MMI), an antithyroid drug commonly used for the treatment of hyperthyroidism; is also used to induce hypothyroidism in experimental studies because of its safety and ease of administration. ${ }^{4}$ It causes alterations in thyroid histology like increased mitotic activity leading to increased apoptosis, chromatin condensation, colloid depletion and follicular morphological disruption. ${ }^{5,6}$ These histological alterations induced by methimazole closely resemble human hypothyroid histological picture making it an ideal experimental model. Thyroxine is considered to be the final treatment of hypothyroidism. It is known to regulate thyroglobulin content of follicles, decreases TSH to normal and has antioxidant properties all of which can have an effect on thyroid histology in hypothyroidism. ${ }^{7,8}$ As the incidence of hypothyroidism is increasing, the major adverse effects of chronic thyroxine therapy are arrhythmias, dyspnea, insomnia which call for the exploration of safer natural alternatives. Two medicinal plants, Commiphora mukul and Withania somnifera can serve as a new milestone in the management of hypothyroidism. Animal studies have shown the 
effect of Commiphora mukul and Withania somnifera in enhancing thyroid function when used individually. ${ }^{9,10}$ However no study could be identified which discussed the effect of these phytochemicals on the histological abnormalities caused by methimazole induced hypothyroidism. So, the present study was designed to investigate the role of these plant extracts in reversion of histological abnormalities caused by methimazole back to normal. In addition gold standard thyroxine is also employed for comparison.

\section{MATERIAL AND METHODS}

This experimental study was conducted on 60 healthy female Sprague Dawley albino rats aged 6-7 weeks weighing 150-170 gm were purchased from University of Veterinary and Animal Sciences and (UVAS) University of Health Sciences, (UHS) Lahore. The rats were housed in standard polypropylene cages in a controlled room temperature of $25 \pm 10^{\circ} \mathrm{C}$ and relative humidity 60 $70 \%$ at PGMI, Lahore. They were given standard laboratory diet ad libitum throughout the study duration. ${ }^{11}$ Animals were kept in a 12-hour lightdark schedule $6 \mathrm{am}$ to $6 \mathrm{pm}$ and all experimental testing was performed during the light phase from $9 \mathrm{am}$ to $12 \mathrm{pm}$. Medicines were given daily between $9 \mathrm{am}$ to $10 \mathrm{am}$. One week was given to rats for acclimatization before starting the experiment. Ethical permission was obtained from Ethical Review Board and all efforts were made to minimize animal suffering according to guidelines provided by the ethical committee.

\section{Inclusion Criteria:}

- Healthy female rats (Hypothyroidism is commoner in females ${ }^{10}$

- Disease free

- Rats weighing 150-170 g will be included

- Age 6 weeks old

Exclusion Criteria:

- Pregnant female rats.

Pregnancy was excluded by weighing the rats during acclamatization period (8 days) daily

\section{Plant extracts and medicine:}

Commiphora mukul and Withania somnifera were purchased and verified by PCSIR Laboratories, Lahore. Ethanolic extract of Commiphora mukul and Withania somnifera was prepared using the method described previously. ${ }^{12,13}$ Medicines Methimazole (Neomarcazole) and Thyroxine were bought from Fazal Din Pharmacy.

\section{Experimental setup:}

60 female rats were divided into 6 groups with 10 animals each. The total study duration was 38 days which included time for induction of hypothyroidism and subsequently treatment with plant extracts.

All groups aside of group 1 were administered drugs orally through gastric gavage except thyroxine (S/C) Group 1: served as Healthy Control.

Group 2: the disease control group in which animals were given only methimazole once daily orally in a dose of $60 \mathrm{mg} / \mathrm{kg} / \mathrm{day}$ for 21 days. This high dose of MMI was used to induce hypothyroidism based on an earlier report ${ }^{14}$. In the remaining groups each rat was administered the same dose of MMI daily for 21 days to induce hypothyroidism after which plant extracts were given for the next 15 days in following doses.

Group 3: Commiphora mukul $0.2 \mathrm{~g} / \mathrm{kg} /$ day

Group 4: Withania somnifera $1.4 \mathrm{~g} / \mathrm{kg} /$

Group 5: Combination of Commiphora mukul $0.2 \mathrm{~g} / \mathrm{kg} /$ day + Withania somnifera $1.4 \mathrm{~g} / \mathrm{kg} /$ day Group 6: Only Thyroxine $0.6 \mu \mathrm{g} / 100 \mathrm{gm}$ BW S/C.

Method of sacrifice of rats and thyroid sample collection:

Animals were surgically sacrificed. 4-5 rats were placed in a properly covered glass container containing cotton soaked with chloroform. After ten minutes the rats were completely anaesthetized for organ collection. A vertical midline incision was given to expose the trachea, and then thyroid was identified and taken out.

Preparation of thyroid for light microscopy:

Each lobe of thyroid gland was processed for light microscopy, after isolation. Each thyroid lobe was fixed in $3.7 \%$ phosphate-buffered formalin $(\mathrm{pH}=7.2)$. They were dehydrated through an ethanol series and xylol and then embedded in paraffin. 5 $\mu \mathrm{m}$ thick paraffin sections were stained with hematoxylin/eosin method for general histological analysis. These sections were taken from the anterior, media land posterior part of the thyroid lobe (five non serial sections per each chosen part of a lobe). These sections were then analyzed on light microscope. ${ }^{7}$ Histological evidence of hypothyroidism was classified in terms of the extent of abnormality. The degree of each histological change was graded from 0-3.

- Grade 0 (absent) indicates no significant change in histology,

- Grade 1 (mild) indicates $1-25 \%$ change,

- Grade 2 (moderate) indicates $26-50 \%$ change, and

- Grade 3 (marked) indicates greater than 51\% change. ${ }^{15}$

An experienced pathologist blinded to the study groups examined the sections obtained from thyroid tissues and graded them. 


\section{Histopathological Parameters}
1. Variation in follicles
2. Epithelium
3. Colloid
4. Chromatin
5. Nuclear Pyknosis

\section{Statistical analysis:}

Data was analyzed by SPSS Version 20.0. Comparison of histological parameters was performed using Chi-square test. P-value $<0.05$ is considered statistically significant.

\section{RESULTS}

Follicular morphology, epithelium, colloid content and chromatin were normal in healthy control group. The group which received only MMI and no other treatment showed significant changes in histology such as severe follicular morphological abnormalities which include narrowing of follicles and fusion of follicles. In addition epithelium was also severely disrupted and colloid content depleted. There was severe damage to chromatin leading to condensation and even absence of chromatic along with multiple pyknotic foci. After receiving treatment with plant extracts $\mathrm{CM}$ and WS these changes were reversed to normal in varying degrees as can be seen in following graphs and figures. Details of results are given in graphs below.

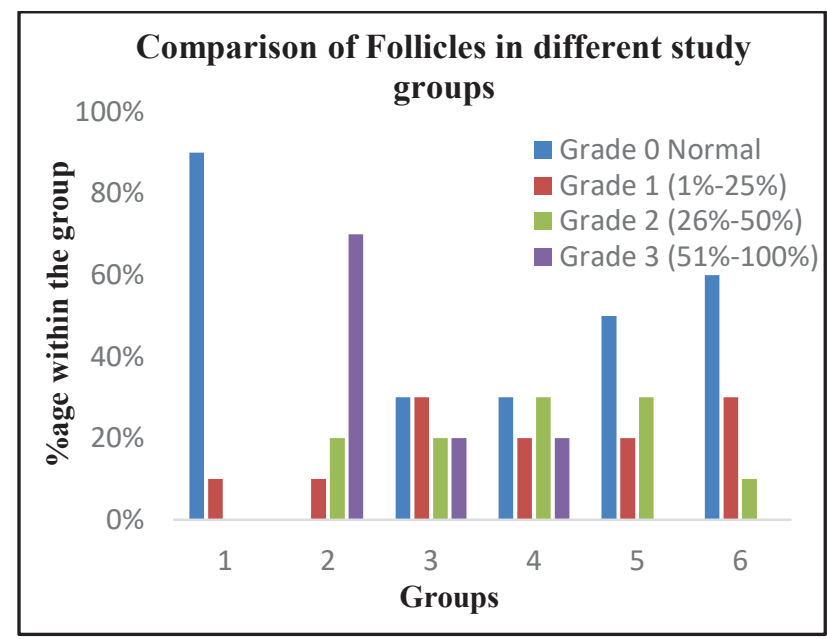

Chi-square $=48.73$

p-value $<0.001^{* *}$

Graph 1: Comparison of Follicles in different study groups

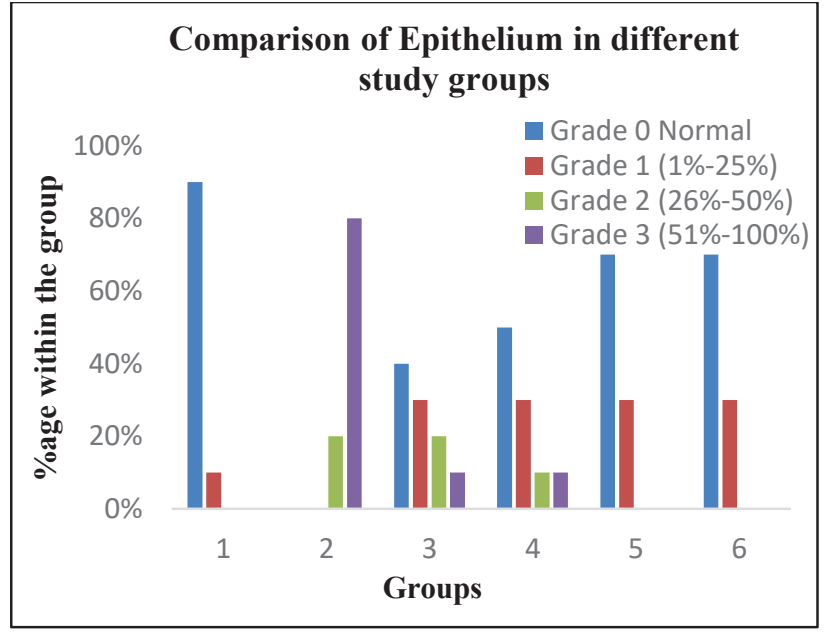

Chi-square $=48.73$

p-value $<0.001^{* *}$

Graph 2: Comparison of Epithelium in different study groups

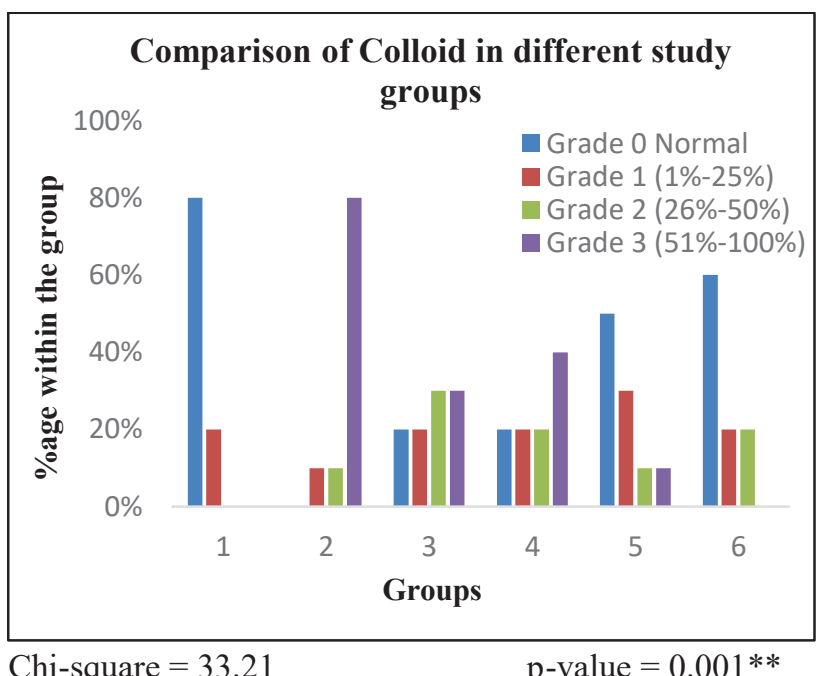

Graph 3: Comparison of Colloid in different study groups

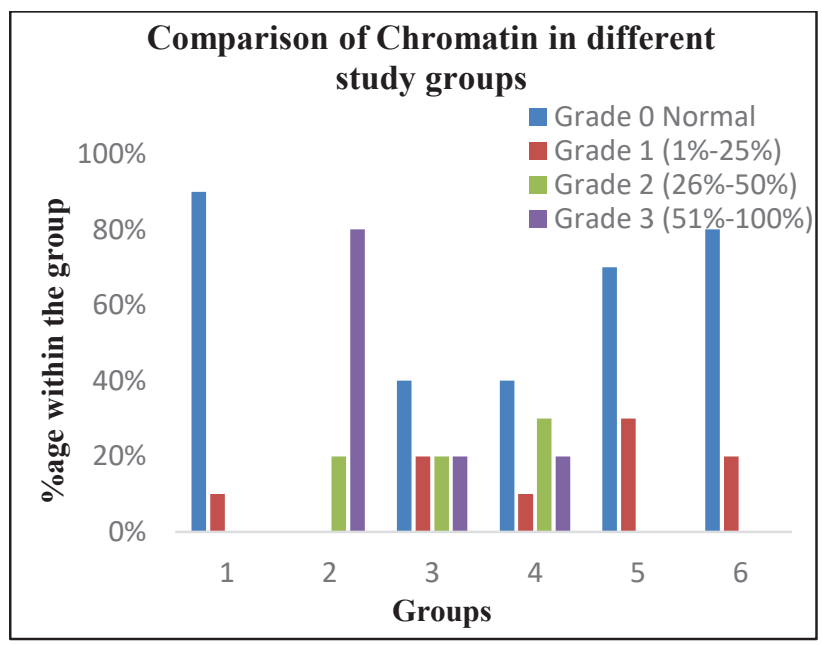

Chi-square $=45.18$

p-value $<0.001 * *$

Graph 4: Comparison of Chromatin in different study groups 


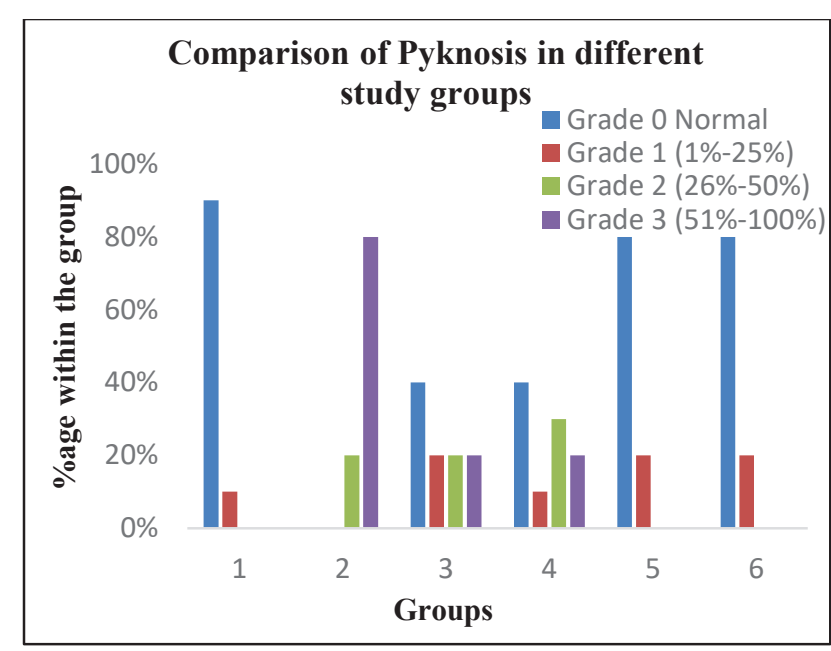

Chi-square $=44.89$ p-value $<0.001 * *$

Graph 5: Comparison of Pyknosis in different study groups

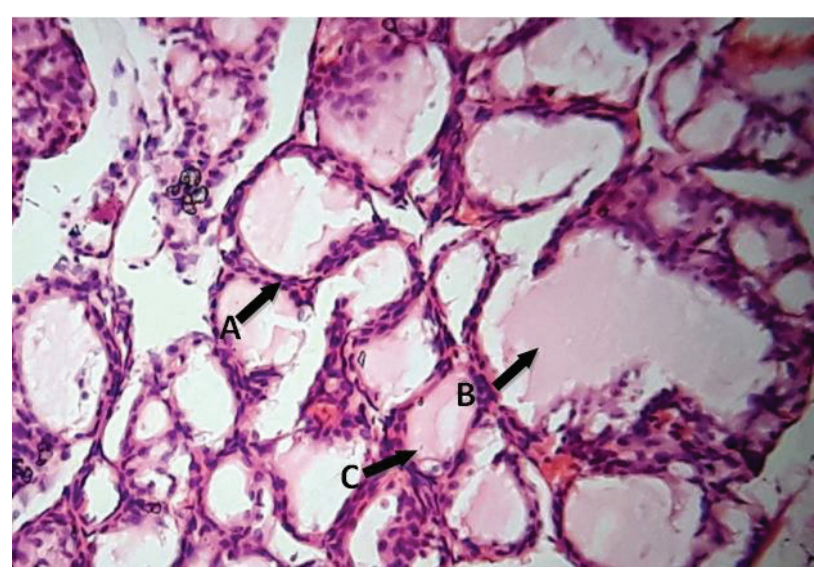

Figure 1: Showing normal thyroid histology (G-1)

$\mathbf{A}=$ Thyroid Epithelium

$\mathbf{B}=$ Colloid present in follicle

C=Normal Follicle

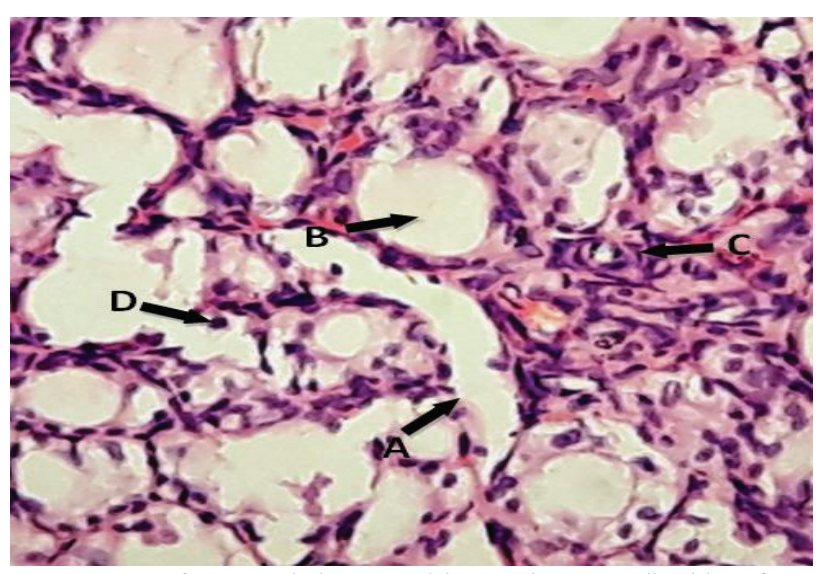

Figure 2: After receiving Methimazole $60 \mathrm{mg} / \mathrm{kg} / \mathrm{day}$ for 21 days $(\mathrm{G}-2)$

$\mathbf{A}=$ Disrupted epithelium

$\mathbf{B}=$ Absent colloid

$\mathbf{C}=$ Follicular disruption

$\mathbf{D}=$ Multiple pyknotic foci

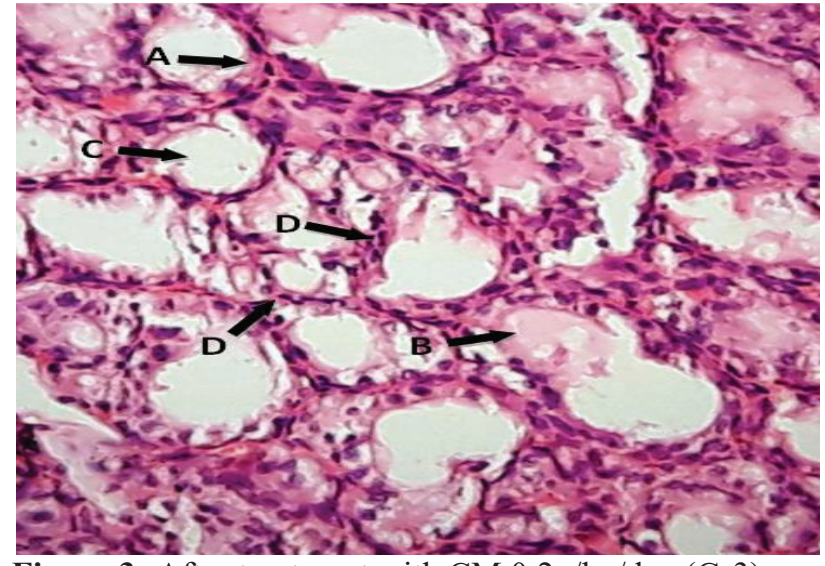

Figure 3: After treatment with CM $0.2 \mathrm{~g} / \mathrm{kg} /$ day (G-3)

$\mathbf{A}=$ Thyroid epithelium not aligned

$\mathbf{B}=$ Some colloid

C $=$ Follicular morphology

$\mathbf{D}=$ Multiple pyknotic foci

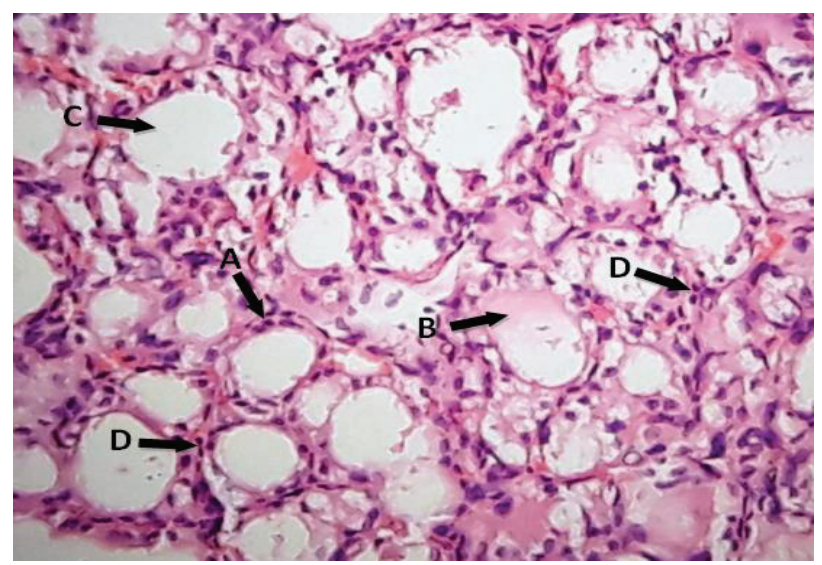

Figure 4: After administering WS $1.4 \mathrm{~g} / \mathrm{kg} /$ day (G-4)

$\mathbf{A}=$ Thyroid epithelium partially aligned

$\mathbf{B}=$ some colloid

$\mathbf{C}=$ Follicular morphology near to normal

$\mathbf{D}=$ Multiple pyknotic foci

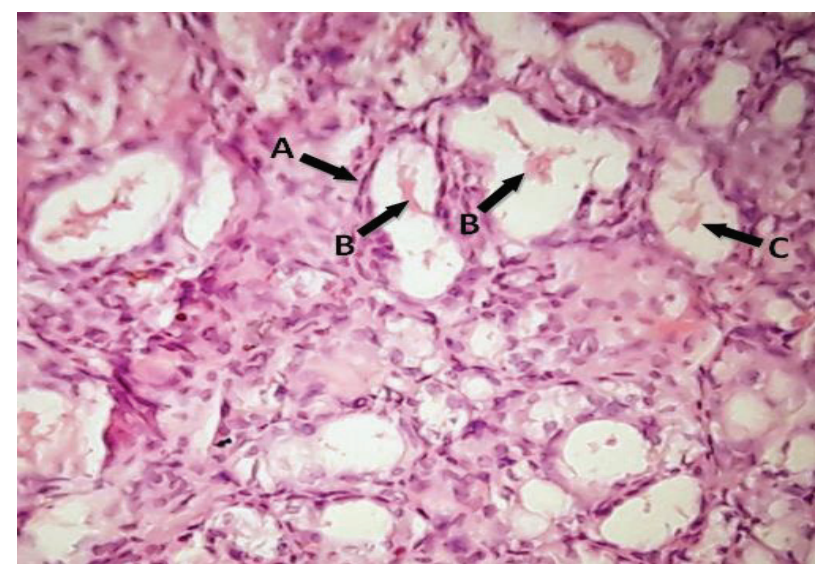

Figure 5: After treatment with CM $0.2 \mathrm{~g} / \mathrm{kg} /$ day+WS

$1.4 \mathrm{~g} / \mathrm{kg} /$ day $(\mathrm{G}-5)$

$\mathbf{A}=$ Follicular epithelium aligning

$\mathbf{B}=$ Colloid present in majority of the follicles

$\mathbf{C}=$ Follicular morphology returning

$\mathbf{D}=$ Focal pyknotic foci, not very prominent 


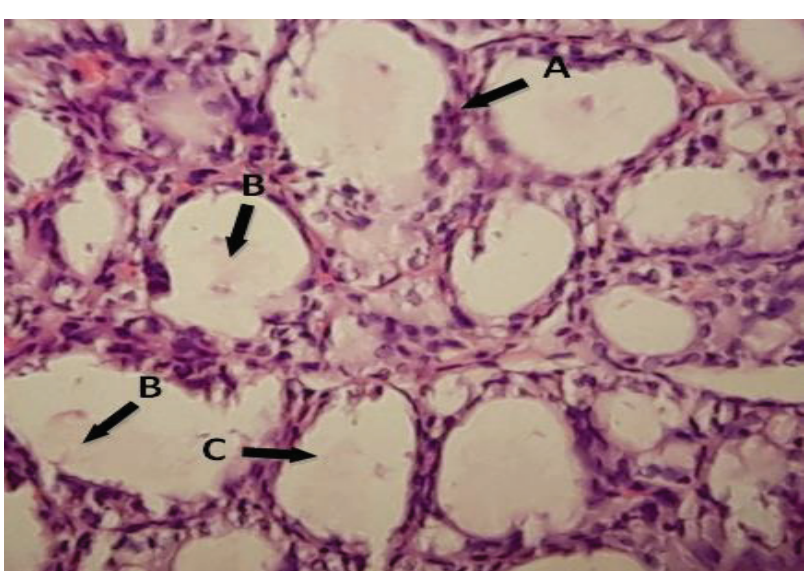

Figure 6:Treated with Thyroxine $0.6 \mu \mathrm{g} / 100 \mathrm{gm}$ BW(G-6) $\mathbf{A}=$ Normal follicular epithelial alignment returning $\mathbf{B}=$ Partially filled follicles with colloid $\mathbf{C}=$ Follicular morphology partially normal $\mathbf{D}=$ Focal pyknotic foci seen, not very prominent

\section{DISCUSSION}

Commiphora mukul and Withania somnifera are two medicinal herbs used for various ailments. Their beneficial role in correcting T3, T4 and TSH levels in hypothyroidism when used together is already ascertained in literature ${ }^{16}$ but their histological impact on thyroid when used alone and in combination is still to be determined. So, our present work is designed to look for the effects of these two plant extracts i.e. ethanolic extract of Commiphora mukul (CMEE) and Ethanolic extract of Withania somnifera (WSEE) on the reversion of histopathological abnormalities caused by methimazole induced hypothyroidism. It will give us an insight regarding the effectiveness of these plant extracts in the management of clinical hypothyroidism thus serving as a potential resource in this domain. A histological analysis of thyroid of all the groups was performed after 38 days of experimentation using a grading system ${ }^{15} .90 \%$ of the animals of G-1 healthy control group had normal follicular morphology, epithelium, chromatin and no pyknosis whereas $80 \%$ animals had normal colloid content. Rest of the groups were viewed in the light of these findings.

Thyroid follicles: G-2, disease control group which was given MMI only for 21 days and left untreated for remaining 15 days exhibited severe follicular disruption with $70 \%$ of the animals having grade 3 follicular derangement. This proved that giving MMI to induce hypothyroidism causes changes in thyroid follicular histology making them irregular and narrowed as also seen in literature ${ }^{5}$. These follicular abnormalities can be explained on the basis of decreased levels of T3, T4 following MMI administration which in turn lead to increased TSH levels by negative feedback mechanism. This increase in TSH level is mainly responsible for all follicular changes ${ }^{5}$. Moving toward the treatment groups, the group $3 \& 4$ which received CMEE \& WSEE respectively showed some recovery of follicles with $30 \%$ animals reverting to normal while the rest of the animals showed mild to moderate degree of follicular damage. Our extracts appeared to have corrected serum T3, T4 and TSH levels to some extent as seen in previous study but it was incomplete 9,10 . The group receiving the combination of both these extracts (CMEE +WSEE) depicted even better results with 50\% animals showing normal follicles and no animal having grade 3 severe disruption. The plausible mechanism could be based upon improvement in T3, T4 \& TSH levels that were deranged by high dose of MMI as evidenced from literature 9,10 . First line drug thyroxine alone showed $60 \%$ normal follicles which was comparable to the group receiving the combination of extracts. Our result shows effectivity of the combination CMEE+WSEE in correcting thyroid functioning and ultimately follicular histology nearly equal to therapeutic effect of thyroxine, the gold standard.

Thyroid epithelium: While going through the epithelial histology of disease control group (G-2) we found that $80 \%$ of the animals had severe epithelial disruptions which were caused by high dose of MMI. As seen in a previous study, $\mathrm{H} 2 \mathrm{O} 2$ is essential for normal thyroid functioning and signaling at low concentrations ${ }^{17}$. MMI when given in such high doses as in our study causes inhibition of G6PD and catalase which in turn imposes oxidative stress due to reactive oxygen species. This shows that $\mathrm{H} 2 \mathrm{O} 2$ which was previously harmless now became toxic causing epithelial disruption. ${ }^{14,}$ ${ }^{17}$. In the group treated with extracts individually, CMEE group 3 \& WSEE group 4; there was partial recovery with only $40 \%$ \& $50 \%$ animals showing normal epithelium respectively while rest of the animals had mild epithelial disruption. This depicts the ability of these plant extracts to counteract the oxidative stress imposed by $\mathrm{H}_{2} \mathrm{O}_{2}$ partially when used individually because of their antioxidant properties ${ }^{18,19}$. To our greatest surprise the group 5 which received the combination CMEE + WSEE and group 6 which received the gold standard thyroxine showed similar results with $70 \%$ animals having normal epithelial histology. This again reinforced the therapeutic usefulness of combining CMEE and WSEE which is comparable to thyroxine and can even replace thyroxine because of their extraordinary ability to counteract oxidative stress when used together ${ }^{8}$. 
Colloid: In the group 2 (disease control) 80\% animals had colloid deficient follicles due to high dose of MMI used. One of the actions of MMI is to inhibit thyroglobulin iodination and literature shows that such goitrogens (MMI) can decrease the iodine content of thyroglobulin ultimately leading to colloid depletion at high doses ${ }^{6}$. Another study also emphasized the role of increased TSH levels in colloid depletion ${ }^{20}$. It may have enhanced expression of thyroid-specific genes required in thyroglobulin production and its breakdown 21 . Looking at the histopathology of treatment groups, CMEE (G-3) \& WSEE (G-4) when used individually showed minimal recovery of colloid with only 2 animals in each group having normal colloid filled follicles. This showed that both these extracts when given alone have some therapeutic effect probably because of their ability to enhance thyroid hormone production as seen in literature which plausibly occurs because of iodination of thyroglobulin and ultimately leading to production of colloid ${ }^{9,10}$. The combination group i.e. group 5 which received CMEE +WSEE and group 6 which was given thyroxine showed close results with $50 \%$ and $60 \%$ animals respectively having normal colloid content. This again demonstrated the beneficial role of full dose combination of extracts which would have worked to normalize colloid content by utilizing the thyrotropic effect of both extracts together.This elucidates the phenomenon of synergism. Thyroxine also increases the colloid content in the follicles by influencing thyroglobulin?

Chromatin: $80 \%$ animals of G-2, disease control group had severe grade 3 damage to chromatin leading to condensation and even absence of normal chromatin which is the result of high dose of MMI also supported by a previous study ${ }^{5}$. This could be explained on the basis of increased mitotic activity in the gland because of elevated serum TSH levels resulting from MMI administration to induce hypothyroidism. It was also seen in literature that such abnormalities in chromatin leads to altered binding affinity of thyroid hormone receptors for T3, the active form; causing abnormalities ${ }^{22}$. After using extracts CMEE \& WSEE individually for 15 days in group 3 and group 4 respectively, animals showed some recovery with only $40 \%$ having normal chromatin. This depicts that these phyto chemicals do have some therapeutic effectiveness when used individually as seen in other parameters as well probably working by normalizing T3, T4 and ultimately lowered TSH levels also evident from literature 9,10 . The lowered TSH probably decreases the mitotic activity and condensation of chromatin but the correction was partial. The combination CMEE +WSEE (G-5) was even better with $70 \%$ animals showing reversion to normal chromatin after which group 6 given thyroxine had $80 \%$ animals with normal chromatin. These two groups did not have significant therapeutic difference which implies that the combination dose is nearly as effective as thyroxine in correcting T3, T4 and TSH levels ultimately leading to normalization of chromatin. In addition, this normal chromatin would plausibly restore the binding affinity of thyroid hormone receptors for the biologically active triiodothyronine T3 making the hypothyroid picture even better but this still needed to be proven.

Pyknosis: $80 \%$ animals in MMI treated disease control group (G-2) had grade 3 multiple pyknotic foci. This severe pyknosis could be explained on the basis of hyperplasia induced by MMI which is caused by frequent mitotic activities due to raised TSH levels. To counteract with these mitotic activities and to maintain homeostasis, apoptotic activities are also increased leading to multiple pyknotic foci ${ }^{5}$. Another reason for this can be explained on the basis of involvement of Fas/FasL system in apoptosis in such thyroid glands ${ }^{23}$. After giving the extracts individually for 15 days animals in group $3 \mathrm{CMEE}$ and group $4 \mathrm{WSEE}$ showed partial recovery i.e. $40 \%$ animals had no pyknosis. This depicts that the increased apoptotic activity imposed by MMI as discussed above is partially corrected by these extracts showcasing their role in treatment of hypothyroidism. Astonishing results were shown by combination of extracts CMEE + WSEE which were also similar to results of thyroxine treated group. There were only focal pyknotic foci in these groups and $80 \%$ animals had no pyknotic changes at all. This emphasizes on the effectivity of this combination in combating hyperplasia induced by high TSH levels (MMI induced). Plausible working mechanism behind this effect is the ability of this combination to decrease TSH levels as much as gold standard thyroxine 16. The effectivity of these extracts in reverting thyroid histology back to normal enables us to infer that these two extracts when given together in above mentioned doses can even substitute gold standard thyroxine hailing it to be a new phytoresource in the treatment of hypothyroidism.

\section{CONCLUSION}

In methimazole induced hypothyroid gland CMEE and WSEE when used individually caused partial recovery of histological abnormalities. However, 
treatment with full dose combination of CMEE $0.2 \mathrm{~g} / \mathrm{kg}+$ WSEE $1.4 \mathrm{~g} / \mathrm{kg}$ orally for 15 days showed reversion of histological characteristics comparable to gold standard thyroxine proving the potential of this combination to substitute thyroxine. Further studies of the CMEE and WSEE combination are required to determine the detailed pharmacodynamics and genomics. These should be followed by clinical trials if possible.

\section{REFERENCES}

1. Unnikrishnan AG, Menon UV. Thyroid disorders in India: An epidemiological perspective. Indian J Endocrinol Metab.2011 Jul;15(Supp12):S78-81

2. Unnikrishnan AG, Kalra S, Sahay R K, Bantwal G, John M, Tewari N. Prevalence of hypothyroidism in adults: An epidemiological study in eight cities of India. Indian J Endocrinol Metab.2013; 17(4):647-52.

3. Mansoor R, Rizvi SSR, Huda ST, Khan C. Spectrum of Thyroid Diseases. Ann Pak Inst Med Sci. 2010; 6(2):101-6.

4. G. S. Argumedo CRS, H. J. Olguín Experimental Models of Developmental Hypothyroidism. Hormone and Metabolic Research. 2012; 44:79-85.

5. Majacakic Milosevic AK, Vukosava Davidovi. Methimazole-induced hypothyroidism in rats: effects on body weight and histological characteristics of thyroid gland. Jugoslov Med Biohem. 2004; 23(2):143-7.

6. Miloni E. Thyroglobulin-Rich Colloid Goitres: A Result of the Combined Action of Lithium and Methimazole on the Rat Thyroid. Acta Endocrinol (Copenh) 1983; 103(2):231-4.

7. Njia M. Ali Rajab MU, Maja Cakic-Milosevic Histological and ultrastructural alterations of rat thyroid gland after short-term treatment with high doses of thyroid hormones. Saudi Journal of Biological Sciences. 2015 (2015):1-9.

8. Siegel HBSM. Antioxidant activity of thyroxine and related substances: Effect on in vitro cell growth. Experimantal cell research. 1968; 49(1)::25-

9. Panda S, Kar A. Guggulu (Commiphora mukul) potentially ameliorates hypothyroidism in female mice. Phytother Res 2005; 19(1):78-80.

10. Panda S, Kar A. Withania somnifera and Bauhinia pupurea in the regulation of circulating thyroid hormone concentrations in female mice. J Ethnopharmacol.1999;67:233239.
11. Lab Diet, laboratory rodent diet page 5001, last updated 19/03/2015 Cited in 2017. Available at www.labdiet.com.

12. Saralakumari BRD. Antihyperglycemic, hypolipidemic and antioxidant activities of ethanolic extract of Commiphora mukul gum resin in fructose-fed male Wistar rats Springer. 2012; 68:573-82.

13. El-Boshy Mel S, Abdalla OM, Risha A, Moustafa F. Effect of Withania somnifera Extracts on Some Selective Biochemical, Hematological, and Immunological Parameters in Guinea Pigs Experimental Infected with E. coli. ISRN veterinary science. 2013:153427.

14. Cano-Europa C,Blass Valdivia V, Methimazole induced hypothyroidism causes alteration of the redox environment,oxidative stress, and hepatic damage; events not caused by hypothyroidism itself. Ann Hepatol 2010; 19(1):80-8.

15. Yuji Mizukami Tm, Akitaka Nonomura, Takuma Hashimoto Nt, Fujitsugu Matsubara, Takazakura Ae. Iodine-Induced Hypothyroidism: A Clinical and Histological Study of 28 Patients. Journal of Clinical Endocrinology and Metabolism. 1993; 76(2): 466-71.

16. Zulfiqar. T, Alam S. S, Jabbar. A. et.al. Effects of Commiphora mukul, Withania somnifera and Thyroxine on Thyroid Profile in Murine Model of Hypothyroidism. Proceedings S.Z.P.G.M.I. 2019: 33(4):25-30.

17. Landex NL TJ, Kayser L. Methimazole increases $\mathrm{H} 2 \mathrm{O} 2$ toxicity in human thyroid epithelial cells. Acta Histochem. 2006; 108(6):431-9.

18. B. Ramesh, D. Saralakumari diabetic rat model Ramesh B, Karuna R, Sreenivasa Reddy S, Ramesh Babu K, Ramatholisamma P, Appa Rao $\mathrm{CH}$, Saralakumari D et al. Antihyperglycemic and antioxidant activities of alcoholic extract of Commiphoramukulgum resin in STZ induced diabetic rats. J Pathophysiol. 2011; 18:255-261.

19. D. A. Barnes RB, P. Singh Nigam and R. Owusu-Apenten. Antioxidant, Anticancer and Antibacterial Activity of Withania somnifera Aqueous Root Extract. Journal of Advances in Biology \& Biotechnology ISSN: 2016; 5(1):1-6.

20. Hans Gerber HS, Angelo Conti, Hanna Engler, Heinz Kohler, Haeberli AA. Reaccumulation of Thyroglobulin and Colloid in Rat and Mouse Thyroid Follicles during Intense Thyrotropin Stimulation A Clue to the Pathogenesis of Colloid Goiters; J Clin Invest. 1981;68:1338-47.

21. Koichi Suzuki AK, Aya Yoshihara. Role of thyroglobulin on negative feedback 
autoregulation of thyroid follicular function and growth; Journal of Endocrinology 2011; 209:169-74.

22. Norman L. Eberhardt Jcr, Lorin K. Johnson. Regulation of activity of chromatin receptors for thyroid hormone: Possible involvement of histone-like proteins (ligand-binding specificity) Proc Nati Acad Sci USA. 1979; 76(10):5005-9.

23. Tamura M, Kimura H, Koji $\mathrm{T}$, Tominaga $\mathrm{T}$, Ashizawa K, Kiriyama $\mathrm{T}$, et al. Role of apoptosis of thyrocytes in a rat model of goiter. A possible involvement of Fas system. Endocrinology 1998; 139, 3646-53.

\section{The Authors:}

Dr. Tehreem Zulfiqar

Assistant Professor,

Department of Pharmacology,

Nishtar Medical University, Multan.
Prof. Saadia Shahzad Alam

Head, Department of Pharmacology,

Shaikh Zayed Medical Complex, Lahore

Dr. Abdul Jabbar

Head, Department of Pharmacology,

Nishtar Medical University, Multan.

Dr. Mariyam Iftikhar

Assistant Professor,

Department of Pharmacology,

Akhtar Saeed Medical and Dental College, Lahore.

\section{Corresponding Author:}

Dr. Tehreem Zulfiqar

Assistant Professor,

Department of Pharmacology,

Nishtar Medical University, Multan.

E-mail: tehreemzulfiqar.tz@gmail.com 